\title{
HELMINTOS PARASITOS DE Rhamdia quelen (QUOY \& GAIMARD, 1824) NO SUL DO BRASIL
}

\author{
DIAS, Joziani Scaglioni ${ }^{1}$; \\ POZZA, Alice ${ }^{1}$; \\ PESENTI, Tatiana Cheuiche ${ }^{1}$; \\ PEREIRA JR., Joaber ${ }^{2}$; \\ Recebido: 06/04/2015 \\ BERNE, Maria Elisabeth Aires ${ }^{3}$.
}

Aceito: $15 / 12 / 2015$

${ }^{1}$ Programa de Pós-Graduação em Parasitologia, Instituto de Biologia, UFPEL; ${ }^{2}$ Professor Doutor, Instituto de Ciências Biológicas, FURG; ${ }^{3}$ Professora Doutora, Instituto de Biologia, UFPEL.

\section{RESUMO}

$\mathrm{R}$ hamdia quelen é um peixe de água doce com hábito alimentar onívoro. Ocorre desde o sudeste do México até o sul da Argentina. São peixes que apresentam boa adaptação em viveiros de piscicultura, com excelente aceitação no mercado consumidor. Com o aumento da aquicultura no Brasil e no mundo, o estudo sobre parasitos de peixes tem aumentado, pois além de causarem mortalidade dos peixes, algumas espécies apresentam potencial zoonótico. O objetivo do trabalho foi identificar parasitos e descrever os locais de infecção destes em $R$. quelen comercializados no sul do Brasil. Foram necropsiados 50 espécimes adquiridos em peixarias, utilizando técnicas específicas para coleta e identificação de parasitos. A prevalência de parasitismo foi de $64 \%(32 / 50)$, sendo $50 \%$ por parasitos da classe Digenea. Clinostomum complanatum o mais prevalente $(40 \%)$, seguido de Tylodelphys cf. destructor (10\%), Acanthostomum sp. (8\%) e Genarchella sp. (4\%). A ocorrência desses parasitos foi maior na musculatura, seguido de gônadas e coração. A prevalência de Nematoda foi de $42 \%$, sendo identificadas larvas de Contracaecum sp. em $24 \%$ e Hysterothylacium sp. em $22 \%$ dos peixes. 0 órgão mais parasitado foi o intestino, seguido do estômago. Diante dos resultados, conclui-se que o elevado parasitismo encontrado em $R$. quelen comercializado em Pelotas apresenta significativo potencial zoonótico, advindo dos helmintos $C$. complanatum e Contracaecum. Sendo assim, alerta-se para os riscos da ingestão deste peixe cru ou mal-cozido, bem como a perdas econômicas à piscicultura advindas da infecção de $R$. quelen por esses parasitos.

Palavras-chave: Peixe. Digenea. Nematoda. Potencial zoonótico. 


\section{INTRODUÇÃO}

Rhamdia quelen (Quoy \& Gaimard, 1824), conhecido popularmente por jundiá, tem distribuição neotropical, ocorrendo desde o sudeste do México até o centro da Argentina (SILFVERGRIP, 1996). Com hábito noturno e comportamento bentônico, prefere locais calmos e profundos dos rios (GOMES et al., 2000).

O seu desenvolvimento ocorre em diferentes ambientes, possuindo hábito alimentar onívoro, alimentando-se preferencialmente de peixes, crustáceos, insetos, restos vegetais e detritos orgânicos (GOMES et al., 2000; GUEDES, 1980).

Por serem peixes adaptados a diferentes ambientes, espécies de Rhamdia vêm apresentando bons resultados em viveiros de piscicultura (GUEDES, 1980), com excelente aceitação no mercado consumidor (CARNEIRO; MIKOS, 2005). Na região sul do Brasil, profissionais da piscicultura têm demonstrado grande interesse pelo $R$. quelen, por seu crescimento acelerado, inclusive nos meses mais frios, característica comum da espécie (CARNEIRO et al., 2002).

Dentre as doenças que afetam $R$. quelen estão as parasitoses, que além dos efeitos deletérios per si, podem estar envolvidas na transmissão de bactérias e/ou vírus responsáveis por outras patologias que trarão reflexos negativos à piscicultura. Essas patologias ocasionam redução nas taxas de assimilação e crescimento dos hospedeiros, podendo provocar diminuição do valor comercial do peixe ou limitar a exploração de algumas espécies (EIRAS, 1994).

O estudo do parasitismo em peixes de interesse comercial é relevante, não somente pelos prejuízos que causa na exploração, mas também pelo potencial zoonótico de muitas espécies. Algumas dessas zoonoses, como Anisakidose, Capilariose e Gnatostomiose, são consideradas emergentes (MC CARTHY; MOORE, 2000).

Dentre os grupos de parasitos registrados em $R$. quelen no Brasil e em outros países, a classe Digenea alberga a maior diversidade, sendo citados: Acanthostomum gneri (Szidat, 1954), na Argentina (LUNASCHI, 1986) e Phyllodistomum rhamdiae descrito por Amato e Amato (1993) no Rio de Janeiro. Metacercárias de Diplostomum (Austrodiplostomum) compactum (Lutz, 
1928), Clinostomum marginatum (Rudolphi, 1819) e Clinostomum heluans (Braun, 1899) foram registrados em Minas Gerais (MOREIRA, 2000). No Rio Grande do Sul, estudo realizado por Morais (2005) relatou metacercárias de C. complanatum, Tylodelphis destructor (Szidat; Nani, 1951), Genarchella sp. e Acanthostomum sp. nesse hospedeiro.

A maioria dos digenéticos é parasito de vertebrados quando adultos. Os peixes podem estar envolvidos como segundo hospedeiro intermediário, ao albergar metacercárias, ou como hospedeiro definitivo, quando parasitados por formas adultas (BUSH et al., 2001). Dentre os digenéticos de peixes, Clinostomum sp. vem sendo alvo de estudos, pois suas metacercárias são encontradas em vários órgãos, causando patologias e mudanças de comportamento, com consequentes perdas econômicas em cultivos (MITCHELL, 1995).

A presença de Clinostomum sp. na musculatura é responsável por degeneração nas fibras musculares, devido a reação inflamatória (SÃO CLEMENTE et al., 1998). A sintomatologia está associada ao local de fixação da metacercária, assim, os cistos grandes, localizados em nadadeiras, dificultam a locomoção e consequentemente a busca por alimentos (PAVANELLI et al., 1998).

Além dos prejuízos econômicos, estudos atentam ao potencial zoonótico, principalmente de C. complanatum (KITAGAWA et al., 2003), que parasita o esôfago de aves piscívoras, seus hospedeiros definitivos. Os peixes e os moluscos desempenham papel de hospedeiros intermediários, apresentando metacercárias encistadas na musculatura e em diversos órgãos (MITCHELL, 1995). Este trematódeo é associado à laringofaringite parasitária em humanos, sendo que a infecção humana ocorre pela ingestão de peixe cru ou mal-cozido que estejam infectados com metacercárias (KAMO et al., 1962).

Casos de infecção humana por C. complanatum foram reportados no Japão (HARA et al., 2014; YAMASHITA, 1938), Israel (WITENBERG, 1944), Índia (CAMERON, 1945), Tailândia (TIEWCHALOERN et al., 1999) e Coreia (PARK et al., 2009).

Embora no Brasil ainda não existam registros de infecção humana por C. complanatum, este parasito tem sido encontrado em várias espécies de peixes (SILVA et al., 2008; SUTILI et al., 2014). No sul do Brasil, observou-se uma prevalência de $64,16 \%$ de $C$. complanatum em $R$. quelen (VIANNA et al., 2005). 
Parasitos nematoides também já foram reportados em outros peixes de água doce (LUQUE et al., 2011; VICENTE; PINTO, 1999). Em R. quelen já foram descritos Procamallanus (Spirocamallanus) hilarii (Vaz \& Pereira, 1934) na Amazônia (BOEGER et al., 2006). Contracaecum sp. em São Paulo (MADI; SILVA, 2005) e Cucullanus pinnai (Travassos, Artigas \& Pereira, 1928) no Rio de Janeiro (VENANCIO et al., 2010).

Nematoides podem causar doenças pela ação espoliativa, tóxica e mecânica, retardando o crescimento e podendo levar à morte. As formas larvais ocasionam perdas econômicas, dificultando a comercialização dos peixes (EIRAS; REGO, 1987).

Dentre os nematoides, os anisakideos, agentes de uma zoonose chamada anisakidose, vem sendo investigada devido ao crescente consumo de peixes crus ou mal-cozidos (UBEIRA et al., 2000). Anisakidose é uma parasitose do trato digestório e caracteriza-se pela fixação na mucosa gástrica de larvas de Anisakidae. É uma zoonose comum no norte da Europa e no Japão, onde é elevado o consumo de peixes crus. Os casos de anisakidose no Japão chegam a 2000 casos por ano, na Europa em torno de 500 e nos Estados Unidos, aproximadamente, 50 casos (AUDICANA et al., 2002).

Considerando os prejuízos econômicos causados pelo parasitismo em peixes cultivados e o potencial zoonótico de algumas espécies, o objetivo deste estudo foi caracterizar a fauna helmíntica e respectivos locais de infecção de parasitos em $R$. quelen comercializados no município de Pelotas, estado do Rio Grande do Sul (RS).

\section{MATERIAL E MÉTODOS}

Entre os anos de 2005 a 2007, foram examinados 50 espécimes de $R$. quelen adquiridos nas peixarias do município de Pelotas (RS), provenientes da Bacia Hidrográfica L40: Piratini-São Gonçalo-Mangueira. Os peixes foram acondicionados em caixas isotérmicas contendo gelo e encaminhados ao laboratório.

Inicialmente foi realizada a análise superficial do corpo, incluindo opérculo e nadadeiras. Após, os órgãos (olhos, brânquias, coração, rim cefálico, bexiga natatória, bexiga urinária, estômago, intestino, fígado, gônadas e baço) foram retirados, individualizados em placas de 
Petri, e tiveram sua superfície, cavidades e conteúdos examinados ao estereomicroscópio. Também foram examinadas, ao estereomicroscópio, a cavidade celomática e a musculatura. Os parasitos encontrados foram fixados em A.F.A. (93\% de álcool a $70{ }^{\circ} \mathrm{GL}, 5 \%$ de formol e 2\% de ácido acético glacial) por 24 horas. Após, os nematoides foram clarificados em lactofenol de Amann, e os digenéticos corados em carmim de Semichon e clarificados em creosoto de Faia, segundo técnica de Amato et al. (1991).

Todos os parasitos foram montados entre lâmina e lamínula para identificação e contagem dos espécimes. A identificação taxonômica foi realizada segundo as chaves de: Yamaguti (1961, 1971), Travassos (1969), Vicente e Pinto (1999), Bray et al. (2008) e Anderson et al. (2009).

Os descritores quantitativos foram calculados conforme Bush et al. (1997).

\section{RESULTADOS E DISCUSSÃO}

Dos 50 espécimes examinados, 32 (64\%) estavam parasitados por pelo menos uma espécie de helminto.

Os parasitos encontrados foram: Clinostomum complanatum (Digenea: Clinostomidae) (Figuras 1A e 1B), Tylodelphys cf. destructor (Digenea: Diplostomidae) (Figura 1C), Acanthostomum sp. (Digenea: Cryptogonimidae) (Figura 1D), Genarchella sp. (Digenea: Hemiuridae) (Figura 1E), Contracaecum sp. (Nematoda: Anisakidae) (Figuras 2A e 2B) e Hysterothylacium sp. (Nematoda: Anisakidae) (Figuras 2C e 2D). 


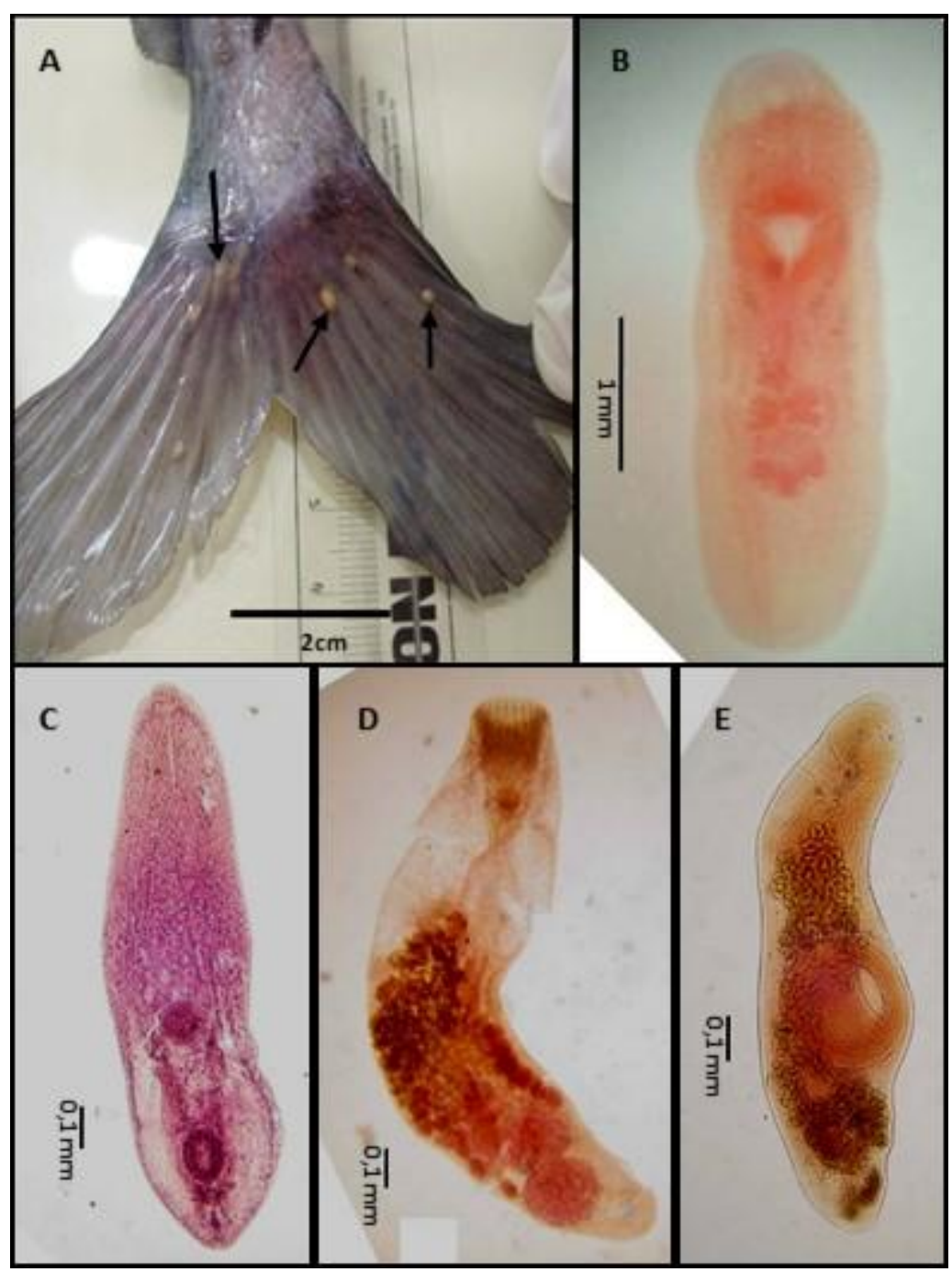

Figura 1- Digenea identificados em Rhamdia quelen comercializados em Pelotas, Rio Grande do Sul. A) Cauda de R. quelen, mostrando os cistos de metacercárias de Clinostomum complanatum. B) Metacercária desencistada de $C$. complanatum. C) Metacercária de Tylodelphys cf. destructor. D) Acanthostomum sp. E) Genarchella sp. 


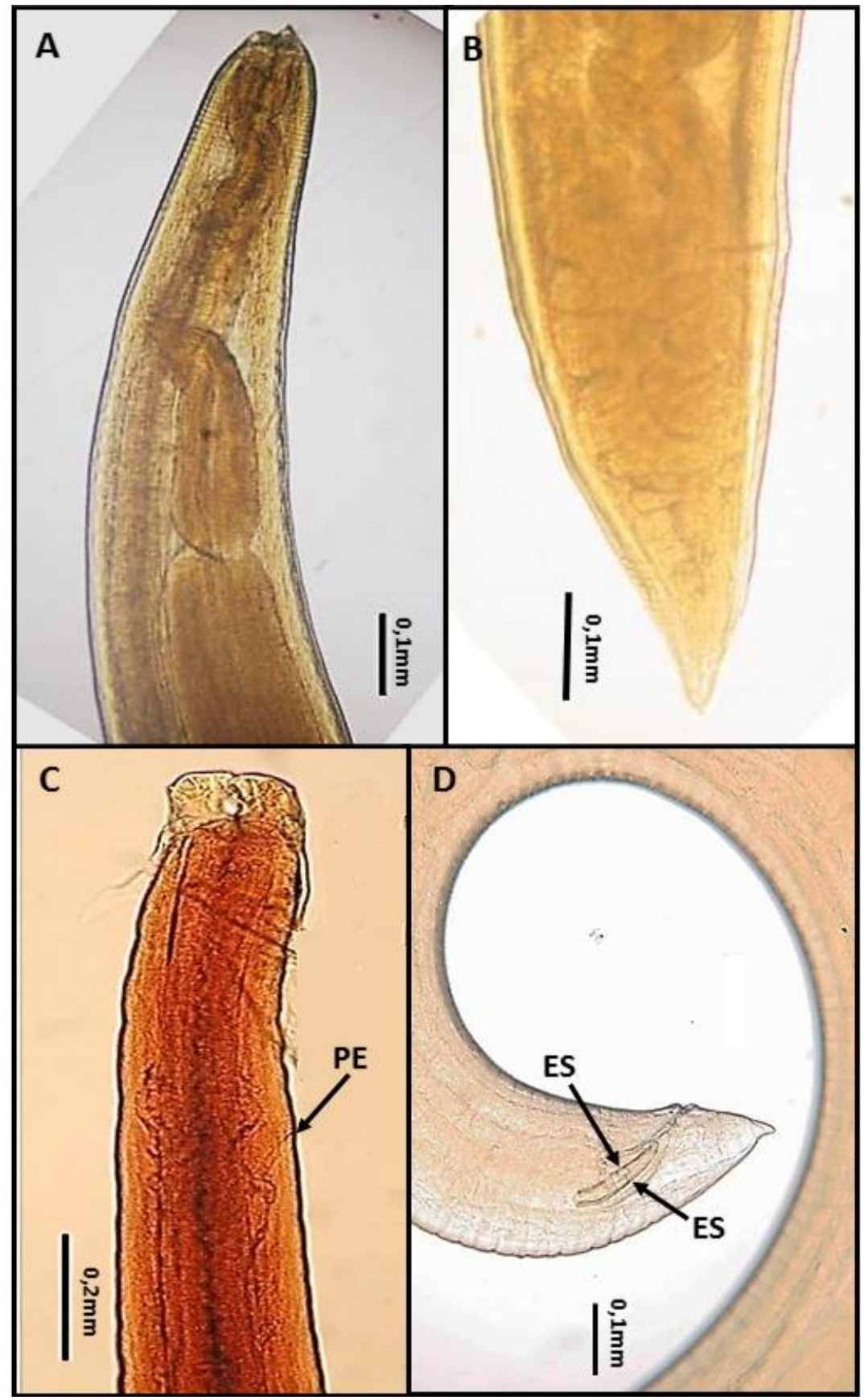

Figura 2 - Nematoda parasitos identificados em Rhamdia quelen comercializados em Pelotas, Rio Grande do Sul. A) Região anterior de larva de Contracaecum sp. B) Região posterior de larva de Contracaecum sp. C) Região anterior de Hysterothylacium sp. mostrando o poro excretor (PE). D) Região posterior do macho de Hysterothylacium sp. mostrando os espículos (ES).

As maiores prevalências observadas foram: C. complanatum (40\%), seguido por Contracaecum sp. (24\%), Hysterothylacium sp. (22\%), T. cf. destructor (10\%), 
Acanthostomum sp. (8\%) e Genarchella sp. (4\%) (Tab. 1). As maiores densidades relativas e intensidades médias de infecção por C. complanatum foram registradas na musculatura, brânquias e nadadeiras (Figura 1A, Tab. 2). Os maiores índices parasitários por $T$. cf. destructor ocorreram nas gônadas e no coração e por Hysterothylacium sp. no intestino e estômago (Tab. 2).

Tabela 1 - Índices parasitológicos de helmintos em Rhamdia quelen $(n=50)$ comercializados em Pelotas, Rio Grande do Sul.

\begin{tabular}{lcccc}
\hline & Positivos & P (\%) & IM & AM \\
\hline DIGENEA & 25 & 50 & 40,68 & 20,34 \\
Clinostomum complanatum & 20 & 40 & 28,75 & 11,5 \\
Tylodelphys cf. destructor & 5 & 10 & 7,04 & 70,4 \\
Acanthostomum sp. & 4 & 8 & 1,5 & 18,75 \\
Genarchella sp. & 2 & 4 & 0,3 & 7,5 \\
NEMATODA & 11 & & & 1,32 \\
Hysterothylacium sp. & 21 & 22 & 3,14 & 0,54 \\
Contracaecum sp. & 12 & 24 & 3,25 & 0,78 \\
\hline P(Prevalência); IM (Intensidade & & & 2,45 & \\
\hline
\end{tabular}

P (Prevalência); IM (Intensidade Média de infecção); AM (Abundância Média). 
Tabela 2 - Densidades relativas e intensidade média por local de infecção, entre parênteses, de helmintos em Rhamdia quelen $(n=50)$ comercializados em Pelotas, Rio Grande do Sul.

\begin{tabular}{|c|c|c|c|c|c|c|c|c|c|c|c|c|c|}
\hline & CC & $M$ & B & $\mathbf{N}$ & 0 & RC & 0 & C & BN & G & $E$ & 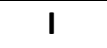 & $\mathbf{F}$ \\
\hline \multicolumn{14}{|l|}{ DIGENEA } \\
\hline $\begin{array}{l}\text { Clinostomum } \\
\text { complanatum }\end{array}$ & - & $\begin{array}{c}464 \\
(23,2)\end{array}$ & $\begin{array}{c}70 \\
(14)\end{array}$ & $\begin{array}{l}30 \\
(6)\end{array}$ & $\begin{array}{c}4 \\
(2)\end{array}$ & $\begin{array}{c}1 \\
(1)\end{array}$ & $\begin{array}{c}1 \\
(1)\end{array}$ & - & - & - & - & - & - \\
\hline $\begin{array}{l}\text { Tylodelphys cf. } \\
\text { destructor }\end{array}$ & $\begin{array}{c}45 \\
(45)\end{array}$ & - & - & - & - & $\begin{array}{c}21 \\
(10.5)\end{array}$ & - & $\begin{array}{l}101 \\
(25)\end{array}$ & $\begin{array}{c}27 \\
(27)\end{array}$ & $\begin{array}{c}156 \\
(156)\end{array}$ & - & $\begin{array}{c}1 \\
(1)\end{array}$ & - \\
\hline Acanthostomum sp. & - & - & - & - & - & - & - & - & - & - & $\begin{array}{c}47 \\
(23,5)\end{array}$ & $\begin{array}{c}28 \\
(9,3)\end{array}$ & - \\
\hline Genarchella sp. & - & - & - & - & - & - & - & - & - & - & $\begin{array}{c}12 \\
(12)\end{array}$ & $\begin{array}{c}3 \\
(3)\end{array}$ & - \\
\hline \multicolumn{14}{|l|}{ NEMATODA } \\
\hline Contracaecum sp. & - & - & - & - & - & - & - & - & - & $\begin{array}{l}10 \\
(5)\end{array}$ & $\begin{array}{c}11 \\
(2,2)\end{array}$ & $\begin{array}{c}17 \\
(2,13)\end{array}$ & $\begin{array}{c}1 \\
(1)\end{array}$ \\
\hline Hysterothylacium sp. & - & - & - & - & - & - & - & - & - & - & $\begin{array}{c}8 \\
(2,67)\end{array}$ & $\begin{array}{c}19 \\
(2,38)\end{array}$ & - \\
\hline
\end{tabular}

Cavidade celomática (CC), musculatura (M), brânquias (B), nadadeiras (N), opérculo (O), Rim cefálico (RC), olho (O), coração $(C)$, bexiga natatória $(B N)$, gônadas $(G)$, estômago $(E)$, intestino $(I)$ e fígado $(F)$.

Neste estudo foi observada maior diversidade de Digenea, com quatro espécies identificadas: C. complanatum, Tylodelphys cf. destructor, Acanthostomum sp. e Genarchella sp., quando comparada à diversidade de Nematoda com duas espécies reportadas: Contracaecum sp. e Hysterothylacium sp. (Tab. 1). Quatro espécies de Digenea também foram identificadas por Morais (2005) em um estudo realizado no sul do Brasil, com $R$. quelen oriundos de piscicultura no município de Arroio Grande (RS) e de ambiente natural no canal São Gonçalo, na divisa dos municípios de Pelotas e Rio Grande (RS), porém não foram encontradas espécies de Nematoda. Segundo Morais (2005), Digenea está entre os grupos de helmintos que albergam maior número de espécies que parasitam $R$. quelen.

As metacercárias de C. complanatum foram detectadas na musculatura, brânquias, nadadeiras, opérculo, rim cefálico e olho de $R$. quelen (Tab. 2). Metacercárias também foram reportadas por Silva et al. (2008) em $R$. quelen provenientes de piscicultura, no município de Santa Maria (RS). Nesse estudo foi observada maior disseminação dos parasitos, sendo 
encontradas metacercárias na cavidade bucal e abdominal, brânquias, olho, miocárdio, intestino, nadadeiras, fígado, estômago e musculatura. A concentração de hospedeiros, como ocorre em cultivos, facilita a disseminação dos parasitos, explicando a maior distribuição dos parasitos nos órgãos reportados por Silva et al. (2008), quando comparado ao presente estudo.

A musculatura foi o sistema com maior número de metacercárias (Tab. 2). Isto se deve à facilidade de penetração da cercária no peixe, através da região do opérculo, migrando para os demais sítios (KALANTAN et al., 1987). Parasitos no músculo podem afetar movimentos, importantes para captura de presas e fuga de predadores, enquanto que nas brânquias diminuem a capacidade respiratória. Quando presentes nas gônadas podem afetar o desempenho reprodutivo e no coração podem comprometer o processo respiratório (HOLMES, 1973). A presença de metacercárias de $C$. complanatum na superfície externa do peixe causa a doença dos pontos pretos ou amarelos, levando a perdas econômicas, visto que dificulta sua comercialização, podendo causar a morte dos hospedeiros jovens e também torná-los mais suscetíveis ao ataque de predadores (PAVANELLI et al., 1998).

No Brasil, R. quelen é um dos peixes mais produzidos na região sul (NETO et al., 2013) com grande aceitação no mercado consumidor (CARNEIRO; MIKOS, 2005). Portanto, o elevado índice de parasitismo na musculatura por $C$. complanatum alerta para o perigo da ingestão de $R$. quelen, em condições inadequadas de cocção, considerando o potencial zoonótico deste parasito com registros de infecção humana (KITAGAWA et al., 2003). Já Acanthostomum sp. e Genarchella sp., registrados no estágio adulto, foram encontrados somente no estômago e intestino (Tab. 2).

Formas adultas de Digenea, com raras exceções, parasitam a luz do aparelho digestório. As localizações atípicas de $T$. cf. destructor e $C$. complanatum observadas principalmente na musculatura, gônadas e coração justificam-se por estes parasitos terem sido encontrados somente na forma de metacercária.

A diversidade parasitária de Nematoda observada em $R$. quelen foi baixa, sendo uma espécie de Contracaecum e uma de Hysterothylacium. A baixa diversidade pode estar relacionada aos sistemas de água doce, que possuem uma biodiversidade menor, por serem 
ecologicamente menos heterogêneos que os ambientes marinhos (MARCOGLIESE, 2001). Ambientes marinhos apresentam uma maior riqueza de invertebrados, hospedeiros intermediários de parasitos com ciclos de vida indireto, aumentando a probabilidade de infecção dos hospedeiros definitivos (DIAS-GRIGÓRIO, 2013).

Dentre os nematoides, os anisakideos apresentam importância em saúde pública, pois são responsáveis por doenças emergentes, como a anisakidose em humanos, doença comum no Japão, com registros na Europa e Estados Unidos, que pode acometer o homem ao consumir peixe cru ou mal-cozido (AUDICANA et al., 2002; FELIZARDO et al., 2009).

Espécies de Contracaecum têm sido reportadas em peixes no Brasil. Martins et al. (2005) listaram 53 espécies de peixes brasileiros, marinhos e de água doce, hospedando espécies desse gênero. Considerando os registros de Madi e Silva (2005) este número sobe para 54 espécies. Estes autores encontraram uma prevalência de $83,37 \%$ e intensidade média de infecção de 28,41 de larvas de Contracaecum sp. em $R$. quelen de um reservatório em São Paulo e Minas Gerais, índices mais altos do que os observados no presente estudo (24\% e $3,25 \%$ respectivamente). Fatores como sazonalidade, qualidade da água e presença de hospedeiros intermediários, influenciam na prevalência de Contracaecum (VERBEL et al., 2006).

Espécies de Hysterothylacium têm sido registradas em peixes de diversos países, porém no Brasil existem poucos registros deste anisakideo. Pereira Jr. et al. (2004) relataram pela primeira vez, no estado do Rio Grande do Sul, larvas de Hysterothylacium em Micropogonias furnieri (Desmarest, 1823) (corvina). A prevalência de 22\% verificada para Hysterothylacium sp., no presente estudo, foi menor que a observada por esses autores, que reportaram estágios larvais desse parasito em 36,89\% de M. furnieri. González (1998), no Chile, e Shih e Jeng (2002), em Taiwan, constataram prevalências maiores em peixes marinhos mantidos em áreas de cultivo, 79\% e 86\%, respectivamente. Esses autores também observaram uma intensidade média de infecção superior $(27,3)$, inclusive com relatos de obstrução intestinal. Neste trabalho, foram observados somente adultos de Hysterothylacium sp. em R. quelen. Outros autores registraram estágios larvais em peixes, como Moravec et al. (1993) que 
observaram larvas deste parasito em peixes de água doce e Pereira Jr. et al. (2004) em peixes marinhos.

O conhecimento sobre o desenvolvimento e transmissão de espécies de Hysterothylacium ainda é escasso. Na fase adulta, normalmente são encontrados no intestino de peixes, as larvas já foram amplamente relatadas nos tecidos de peixes, como também de invertebrados aquáticos, que servem como hospedeiros intermediários (ANDERSON, 2000).

Parasitos do gênero Genarchella já foram reportados em diferentes espécies de peixes das famílias Acentrorhynchidae, Characidae, Cichlidae e Pimelodidae em estudos realizados no Brasil, Argentina e Uruguai (FRANCESCHINI et al., 2013). Metacercárias foram registradas infectando brânquias e adultos o intestino de Hemibrycon surinamensis (Géry, 1962), na Amazônia (HOSHINO et al., 2014). Franceschini et al. (2013) relataram a ocorrência de adultos no estômago de Cichla piquiti (Kullander e Ferreira, 2006) em São Paulo. No presente estudo foram encontrados apenas adultos parasitando o estômago e intestino. Em Rhamdia, este helminto já foi reportado em estudos realizados no Brasil (BRANDÃO, 1977), na Nicarágua (WATSON, 1976) e no México (SCHOLZ; SALGADO-MALDONADO, 1994). Para $R$. quelen o registro foi feito por Morais (2005) no estado do RS, no qual também reportou o intestino e estômago como locais de infecção. Pouco se conhece sobre a biologia ou ciclo de vida deste parasito, porém alguns estudos mostraram que moluscos são seus hospedeiros intermediários (HOSHINO et al., 2014).

\section{CONCLUSÃO}

Os espécimes de Rhamdia quelen comercializados no sul do Brasil apresentaram-se infectados por parasitos que podem afetar o desenvolvimento, o desempenho reprodutivo e levar a morte do hospedeiro, com consequentes perdas econômicas em cultivos.

Dos sete helmintos reportados, os mais prevalentes são metacercárias de Clinostomum complanatum e larvas de Contracaecum, potencialmente zoonóticos. Sendo assim, alerta-se para os riscos da ingestão deste peixe cru ou mal-cozido pela população da região estudada, bem como a perdas econômicas à piscicultura advindas da infecção de $R$. quelen por esses parasitos. 


\title{
HELMINTH PARASITES OF Rhamdia quelen (QUOY \& GAIMARD, 1824) IN SOUTHERN BRAZIL
}

\author{
ABSTRACT
}

$\mathrm{R}$ hamdia quelen is an omnivorous freshwater fish, which occurs from southeastern Mexico to southern Argentina. It is well-adapted to pond conditions and has excellent acceptance in the consumer market. Studies on fish parasites have increased parallel to the corresponding increase of aquaculture in Brazil and worldwide. Some of these parasites have zoonotic potential, apart from causing fish mortality. This study aimed to identify parasites as well as their infection sites in $R$. quelen marketed in southern Brazil. Fifty specimens purchased at fish markets were necropsied, and specific techniques for parasite collection and identification were used. The prevalence of parasitism was $64 \%$ $(32 / 50)$ being $50 \%$ by parasites of Digenea class. Clinostomum complanatum showed the highest prevalence rate (40\%), followed by Tylodelphys cf. destructor (10\%), Acanthostomum sp. (8\%) and Genarchella sp. (4\%). The infection was highest in the muscles, followed by the heart and gonads. The prevalence of Nematoda parasites was also high (42\%). Larvae of Contracaecum sp. were identified in $24 \%$ and Hysterothylacium sp. in $22 \%$ of the fishes. The instestine was the most infected organ, followed by the stomach. This study showed that $R$. quelen marketed in the city of Pelotas is highly parasitized with potential zoonotic species, like $C$. complanatum and Contracaecum. Thus, these results serve as a warning on the risks of raw or undercooked $R$. quelen meat ingestion, as well as to the economic losses resulting from $R$. quelen infection by these parasites.

Keywords: Fish. Digenea. Nematoda. Zoonotic potential.

\section{HELMINTOS PARÁSITOS DE Rhamdia quelen (QUOY \& GAIMARD, 1824) EN EL SUR DE BRASIL}

\section{RESUMEN}

$\mathrm{R}$ hamdia quelen es un pez de agua dulce con hábito alimentar omnívoro. Se encuentra desde el sureste de México hasta el sur de Argentina. Son peces que presentan buena adaptación en viveros de piscicultura, con excelente aceptación en el mercado consumidor. Con el aumento de la acuicultura en Brasil y en el mundo, el estudio sobre parásitos de peces ha aumentado, pues además de causar mortalidad de los mismos, algunas especies presentan potencial zoonótico. El objetivo de este trabajo fue identificar parásitos y describir los locales de infección de estos en $R$. quelen comercializados en el sur de Brasil. Fueron necropsiados 50 especímenes adquiridos en pescadería, utilizando técnicas 
específicas para colecta e identificación de parásitos. La prevalencia fue 64\% (32/50), siendo $50 \%$ por parásitos del classe Digenea. Clinostomum complanatum fue el más prevalente (40\%), seguido de Tylodelphys cf. destructor (10\%), Acanthostomum sp. (8\%) y Genarchella sp. (4\%). La infección fue mayor en la musculatura, seguido de gónadas y corazón. La prevalencia de Nematoda fue de $42 \%$, siendo identificadas larvas de Contracaecum sp. en $24 \%$ y Hysterothylacium sp. en $22 \%$ de los pescados. El órgano más parasitado fue el intestino seguido del estómago. Frente a los resultados se tiene que, $R$. quelen comercializada en Pelotas está parasitada por siete diferentes helmintos, siendo los más prevalentes metacercarias de $C$. complanatum y larvas de Contracaecum, que presentan potencial zoonótico. Por lo tanto, se alerta para los riesgos de la ingestión de este pescado crudo o mal cocido, bien como las pérdidas económicas a la industria pesquera provenientes de la infección de $R$. quelen por esos parásitos.

Palabras clave: Pez. Digenea. Nematoda. Potencial zoonótico.

\section{REFERÊNCIAS}

AMATO, S. B.; AMATO, J. F. R. A new species of Phyllodistomum Braun, 1899 (Digenea: Gorgoderidae) from Rhamdia quelen (Quoy e Gaimard, 1824) (Siluriformes:Pimelodidae). Memórias do Instituto Oswaldo Cruz, v. 88, p. 557-559, 1993.

AMATO, J. F. R.; BOEGER, W. A.; AMATO, S. Protocolos para laboratório, coleta e processamento de parasitos de pescado. UFRJ, Imprensa Universitária, 1991. 81p.

ANDERSON, R. C.; CHABAUD, A. G.; WILLMOTT, S. Keys to the Nematode Parasites of Vertebrates. Oxfordshire: CABI Head Office, 2009. 454p.

ANDERSON, R. C. Nematode Parasites of Vertebrates. Their Development and Transmission. 2. ed. New York: CABI Publishing, 2000. 650p.

AUDICANA, M. T.; ANSOTEGUI, I. J.; CORRES, L. F.; et al. Anisakis simplex: dangerous - dead and alive? Trends in Parasitology, v. 18, p. 20-25, 2002.

BOEGER, W. A.; VIANNA, R. T. Monogenoidea. In: Thatcher, V. E. Amazon Fish Parasites. Pensoft Publishers, 2006. Cap. 3, p. 42-116.

BRANDÃO, D. A. Trematódeos digenéticos de Rhamdia sapo (Valencienes, 1840) jundiá no estuário do Guaíba, Rio Grande do Sul, Brasil. Santa Maria: UFSM, 1977. Dissertação (Mestrado em Zootecnia), Universidade Federal de Santa Maria, 1977.

BRAY, R. A.; GIBSON, D. I.; JONES, A. Keys to the Trematoda. Volume 3. CABI, Natural History Museum, London, 2008. 848p. 
BUSH, A.O.; FERNÁNDEZ, J.C.; ESCH, G.W.; SEED, J.R. Parasitism: The diversity and ecology of animal parasits. Cambridge: Cambridge University Press, 2001. 566 p.

BUSH, A. O.; LAFFERTY, K. D.; LOTZ, J. M.; et al. Parasitology meets ecology on terms: Margolis et al. Revisited. Journal of Parasitology, v. 83, p. 575-583, 1997.

CAMERON, T. W. M. Fish-Carried parasites in Canadá. I. Parasites carried by fresh water fish. Canadiann Journal Comparative Medicine, v. 9, p. 245-254, 1945.

CARNEIRO, P. C. F.; BENDHACK, F.; MIKOS, J.; et al. Jundiá: um grande peixe para a região Sul. Panorama da Aqüicultura, v. 12, p. 41-46, 2002.

CARNEIRO, P. C. F.; MIKOS, J. D. Freqüência alimentar e crescimento de alevinos de jundiá, Rhamdia quelen. Ciência Rural, v. 35, p. 187-191, 2005.

DIAS-GRIGÓRIO, M. K. R. Diversidade parasitária e relação parasito-hospedeiro em Colossoma macropomum e seu híbrido tambatinga cultivados em Macapá, estado do Amapá. Amapá: UNIFAP, 2013. 100p. Dissertação (Mestrado em Biodiversidade Tropical), Universidade Federal do Amapá, 2013.

EIRAS, J. C.; REGO, A. A. The histopathology of Scomber japonicus infection by Nemathobothrium scombrium (Trematoda: Dydymozoidae) and of larval anisakid nematode infections in the liver of Pagrus pagrus. Memorias do Instituto Osvaldo Cruz, v. 71, p. 199202, 1987.

EIRAS, J. C. Elementos de Ictioparasitologia. Porto, Portugal: Fundação Eng. António de Almeida, 1994. 339p.

FELIZARDO, N. N.; MENEZES, R. C.; TORTELLY, R.; et al. Larvae of Hysterothylacium sp. (Nematoda: Anisakidae) in the sole fish Paralichthys isoceles Jordan, 1890 (Pisces: Teleostei) from the littoral of the state of Rio de Janeiro, Brazil. Veterinary Parasitology, v. 166, p. 175177, 2009.

FRANCESCHINI, L.; ZAGO, A. C.; ZOCOLLER-SENO, M. C.; et al. Endohelminths in Cichla piquiti (Perciformes, Cichlidae) from the Paraná River, São Paulo State, Brazil. Revista Brasileira de Parasitologia Veterinária, Jaboticabal, v. 22, p. 475-484, 2013.

GOMES, L. C.; GOLOMBIESKI, J. I.; GOMES, A. R. C; et al. Biologia do jundiá Rhamdia quelen (Teleostei, Pimelodidae). Ciência Rural, Santa Maria, v. 30, p. 179-185, 2000.

GONZÁLEZ, L. The life cycle of Hysterothylacium aduncum (Nematoda: Anisakidae) in Chilean marine farms. Aquaculture, v. 162, p. 173-186, 1998.

GUEDES, D. S. Contribuição ao estudo da sistemática e alimentação do Jundiá (Rhamdia sp.) na região central do Rio Grande do Sul. Santa Maria: UFSM, 1980. Dissertação 
(Mestrado em Zootecnia), Curso de Pós-Graduação em Zootecnia, Universidade Federal de Santa Maria, 1980.

HARA, H.; MIYAUCHI, Y.; TAHARA, S.; et al. Human laryngitis caused by Clinostomum complanatum. Nagoya Journal of Medical Science. v. 76, p. 181-185, 2014.

HOLMES, J. C. Site selection by parasitic helminths: interspecific interactions, site segregation, and their importance to the development of helminth communities. Canadian Journal of Zoology, v. 51, p. 333-347, 1973.

HOSHINO, M. D. F. G.; HOSHINO, E. M.; TAVARES-DIAS, M. First study on parasites of Hemibrycon surinamensis (Characidae), a host from the eastern Amazon region. Revista Brasileira de Parasitologia Veterinária, Jaboticabal, v. 23, p. 343-347, 2014.

KALANTAN, A. M.; ARFIN, M.; NIZAMI, W. A. Seasonal incidence and pathogenicity of the metacercariae of Clinostomum. Journal of Parasitology, v. 36, p. 17-23, 1987.

KAMO, H.; OGINO, K.; HATSUSHIKA, R. A unique infection of man with Clinostomum sp., a small Trematoda causing acute lariyngitis. Yonago Acta Medica, v. 6, p. 37-40, 1962.

KITAGAWA, N.; ODA, M.; TOTOKI, T.; et al. Lidocaine apray used to capture a live Clinostomum complanatum parasite causing human laryngitis. American Journal of Otolaryngology, v. 24, p. 341-343, 2003.

LUNASCHI, L. Helmintos parasitos de peces de agua dulce de la Argentina. V. Redescripcion de Acanthostomum (Atrophocaecum) gnerii (sic) Szidat, 1954 (Trematoda: Acanthostomidae). Neotropica, v. 32, n. 87, p. 35-42, 1986.

MADI, R. R.; SILVA, M. S. R. Contracaecum Railliet \& Henry, 1912 (Nematoda, Anisakidae): o parasitismo relacionado à biologia de três espécies de peixes piscívoros no reservatório do Jaguari, SP. Revista Brasileira de Zoociência, v. 7, p. 15-24, 2005.

MARCOGLIESE, D. J. Pursuing parasites up the food chain: implication of food web structure and function on parasite communities in aquatic systems. Acta Parasitologica, v. 46, p. 8293, 2001.

MARTINS, M. L.; ONAKA E. M.; FENERICK, J. JR. Larval Contracaecum sp. (Nematoda: Anisakidae) in Hoplias malabaricus and Hoplerythrinus unitaeniatus (Osteichthyes: Erythrinidae) of economic importance in marshlands of Maranhão, Brazil. Veterinary Parasitology, v. 127, p. 51-59, 2005.

MCCARTHY, J.; MOORE, T. A. Emerging helminth zoonoses. International Journal for Parasitology, v. 30, p. 1351-1360, 2000. 
MITCHELL, A. J. Yellow grubs and other problems associated with aquatic birds. Aquaculture Magazine, v. 21, p. 93-97, 1995.

MORAIS, N. C. M. Helmintos parasitos de jundiá, Rhamdia quelen (Quoy \& Gaimard, 1824) (Siluriformes) coletados em ambiente natural e em estação de piscicultura no sul do RS. Pelotas: UFPEL, 2005. 70p. Dissertação (Mestrado em Veterinária), Faculdade de Veterinária, Universidade Federal de Pelotas, 2005.

MORAVEC, F.; KOHN, A.; FERNANDES, B. M. M. Nematode parasites of the Paraná River, Brazil. Parte 2. Seuratoidea, Ascaridoidea, Habronematoidea and Acuarioidea. Folia Parasitológica, v. 40, p. 115-134, 1993.

MOREIRA, N. I. B. Helmintos parasitos de peixes de lagos do médio Rio Doce, Minas Gerais, Brasil. Belo Horizonte: UFMG, 2000. 193p. Tese de doutorado (Doutorado em Parasitologia) Curso de Pós-Graduação em Parasitologia, Universidade Federal de Minas Gerais, 2000.

NETO, P. G. B.; DUTRA, F. M.; BALLESTER, E. L. C.; et al. Crescimento e sobrevivência de larvas do jundiá, Rhamdia quelen, alimentadas com alimento vivo enriquecido e dieta artificial. A Revista Brasileira de Ciência Veterinária, v. 20, p. 216-221, 2013.

PAVANELLI, G. C.; EIRAS, J. C.; TAKEMOTO, R. M. Doenças de Peixes - Profilaxia, Diagnóstico e Tratamento. Maringá: EDUEM-Editora da Universidade Estadual de Maringá, 1998. 264p.

PARK, C. H.; KIM, J. S.; JOO, H. S. A human case of Clinostomum complanatum infection in Korea. Korean Journal Parasitology, v. 47, p. 401-404, 2009.

PEREIRA JUNIOR, J.; FRANCIS, A. M.; MORAIS, N. C. M.; et al. Hysterothylacium sp. Larvae (Nematoda: Anisakidae) in Micropogonias furnieri (Sciaenidae) from Rio Grande do Sul coast, Brazil. Atlântica, v. 1, p. 55-60, 2004.

SÃO CLEMENTE, S. C.; MATOS, E.; TORTELLY, R.; et al. Histopatologia do parasitismo por metacercárias de Clinostomum sp. em Tamoata hoplosternum littorale (Hancock, 1828), Parasitología al día, v. 22, p. 1-2, 1998.

SATO, M. C. B.; PAVANELLI, G. C. Digenea de Pimelodus maculatus (Osteichthyes, Pimelodidae) das bacias dos rios São Francisco e Paraná, Brasil. Parasitología Latinoamericana, v. 59, p. 123-131, 2004.

SCHOLZ, T.; SALGADO-MALDONADO, G. On Genarchella isabellae (Digenea: Derogenidae) from Cichlid and Pimelodid fishes in Mexico. Journal of Parasitology, v. 80, p. 1013-1017, 1994.

SHIH, H. S.; JENG, M. S. Hysterothylacium aduncum (Nematoda: Anisakidae) Infecting a Herbivourous Fish, Siganus fuscencens, of the Taiwanese Coast of the Nortwest Pacific. Zoological Studies, v. 4, n. 2, p. 208-215, 2002. 
SILFVERGRIP, A. M. C. A systematic revision of the neotropical catfish genus Rhamdia (Teleostei, Pimelodidae). Stockolm: SU, 1996. 156p. Ph.D. Thesis, Departament of Zoology, Stockolm University and Departament of Vertebrate Zoology, Swedish Museum of Natural History, 1996.

SILVA, A. S.; MONTEIRO, S. G.; DOYLE, R. L.; et al. Ocorrência de Clinostomum complanatum em diferentes espécies de peixes de uma piscicultura do município de Santa Maria-RS. Veterinária e Zootecnia, v. 15, p. 27-32, 2008.

SUTILI, F. J.; GRESSLER, L. T.; PELEGRINI, L. F. V. Clinostomum complanatum (Trematoda, Digenea): a parasite of birds and fishes with zoonotic potential in southern Brazil. A Review. Revista Brasileira de Higiene e Sanidade Animal, v. 8, p. 99-114, 2014.

TIEWCHALOERN, S. O.; UDOMKIJDECHA, S.; SUVOUTTHO, S. Clinostomum, trematode from human eye. Southeast Asian. Journal of Tropical Medicine and Public Health, v. 30, p. 382384, 1999.

TRAVASSOS, L.; TEIXEIRA DE FREITAS, J. F.; KOHN, A. Trematódeos do Brasil. Memórias do Instituto Oswaldo Cruz, Rio de Janeiro, 1969. 886p.

UBEIRA, F. M.; VALINAS, B.; LORENZO, S.; et al. Anisaquiois y alergia. Um estúdio soroepidemiológico em la comunidad Autônoma Gallega. Documentos técnicos de Salud Publica, Série B, n. 24, Espana, 2000. 102p.

VENANCIO, A. C. P.; AGUIAR, G. R.; LOPES, P. S.; et al. Metazoan parasites of Mandi-amarelo Pimelodus maculatus and of Jundiá Rhamdia quelen (Osteichthyes: Siluriformes) of Paraíba do Sul River, Volta Redonda, Rio de Janeiro. Revista Brasileira de Parasitologia Veterinária, v. 19, p. 157-163, 2010.

VERBEL, J. O.; ÁVILA, R. B.; FERNANDEZ, J. G. Contracaecum sp. infection in Hoplias malabaricus (Moncholo) from Rivers and marshes of Colombia. Veterinary Parasitology, $v$. 140, p. 90-97, 2006.

VIANNA, R. T.; PEREIRA JR, J.; BRANDÃO, D. A. Clinostomum complanatum (Digenea, Clinostomidae) density in Rhamdia quelen (Siluriformes, Pimelodidade) from South Brazil. Brazilian Archives of Biology and Technology, v. 48, p. 635-642, 2005.

VICENTE, J. J.; PINTO, R. M. Nematóides do Brasil. Nematóides de peixes. Atualização: 19951998. Revista Brasileira de Zoologia, v. 16, p. 561-610, 1999.

WATSON, D. E. Digenea of fishes from the lake Nicaragua. In: THORSON, T. B. Investigation of the icthyophauna lakes. Lincoln, Scholl Life Sciences University of Nebraska, 1976. p. 251260. 
WITENBERG, G. What is the cause of the parasitic laringo-pharyngitis in the Near East (“Halzoun”)? Acta Medica Orientalia, v. 3, p. 191-192, 1944.

YAMAGUTI, S. Systema Helminthum. The Nematodes of Vertebrates. V. III. New York: Interscience Publishers, Inc. Ed, 1961. 679p.

YAMAGUTI, S. Synopsis of digenetic trematodes of vertebrates. V. I and II. Tokio: Keigaku Pub. Co., 1971. 698p.

YAMASHITA, J. Clinostomum complanatum, a trematode parasite new to man. Annotationes. Zoologicae Japonenses, v. 17, p. 563-566, 1938.

Autor para correspondência: Joaber Pereira Júnior. Laboratório de Biologia de Parasitos de Organismos Aquáticos, Avenida Itália, Campus Carreiros, Prédio 6, sala 8G, FURG, Rio Grande (RS), CEP 96650-900, CP. 474. ictiopar.jpjr@gmail.com 